\title{
Baroclinic instability over topography: Unstable at any wave number
}

\author{
by Joseph Pedlosky ${ }^{1,2}$
}

\begin{abstract}
The instability of an inviscid, baroclinic vertically sheared current of uniform potential vorticity, flowing along a uniform topographic slope, becomes linearly unstable at all wave numbers if the flow is in the direction of propagation of topographic waves. The parameter region of instability in the plane of scaled topographic slope versus wave number then extends to arbitrarily large wave numbers at large slopes.

The weakly nonlinear treatment of the problem reveals the existence of a nonlinear enhancement of the instability close to one of the two boundaries of this parametrically narrow unstable region. Because the domain of instability becomes exponentially narrow for large wave numbers, it is unclear how applicable the results of the asymptotic, weakly nonlinear theory are given that it must be limited to a region of small supercriticality.

This question is pursued in that parameter domain through the use of a truncated model in which the approximations of weakly nonlinear theory are avoided. This more complex model demonstrates that the linearly most unstable wave in the narrow wedge in parameter space is nonlinearly stable and that the region of nonlinear destabilization is limited to a tiny region near one of the critical curves rendering both the linear and nonlinear growth essentially negligible.

Keywords: topography, coastal, coastal waves, non linear, slope, wave propagation, most unstable, asymptotic theory, potential vorticity
\end{abstract}

\section{Introduction}

The problem of the instability of coastal currents has a long history (see, e.g., Barth 1989 and Brink 2012 and references therein). Part of the fascination of the problem lies in the effects that topography has on the instability. The interplay between classic baroclinic instability and topographic wave dynamics results in some nonintuitive results that were first discussed by Blumsack and Gierasch (1972) in the context of linear stability theory. I will briefly review their work in the next section, but the essential result is a surprising one. When the normally stabilizing effect of sloping topography is added to the classic model

1. Woods Hole Oceanographic Institution, Woods Hole, MA 02543.

2. Corresponding author: e-mail:jpedlosky@whoi.edu

(C) 2016 Joseph Pedlosky. 
of Eady (1949), the short wave cutoff in that problem is eliminated, and all wave numbers in this inviscid model become unstable when the direction of the shear coincides with the direction of propagation of topographic Rossby waves. Since in the model the flow is in geostrophic balance, this is equivalent to the condition that the slope of the bottom has the opposite sign as the slope of the isopycnals of the shear flow.

The unstable domain in the parameter space delineated by the critical value of the ratio of those slopes extends to all wave numbers but becomes increasingly narrow for large wave numbers. Furthermore, the fact that in the region between the two boundaries of this narrow space of instability, the growth rate has a maximum implies that moving from one of the boundaries into the parametric interior of the region by decreasing the shear enhances the instability. This suggests that if the effects of nonlinearity attributable to the instability reduce the shear, as expected for baroclinic instability, the result could be a nonlinear destabilization. Such an effect was observed in a simple two-layer model by Steinsaltz (1987) who used weakly nonlinear theory. However, for large wave numbers the vertical scale of the perturbation becomes less than the finite layer thickness in the two-layer model leading to questions about the validity of the result. Also, weakly nonlinear theory is limited to an asymptotically small region near the marginal stability curve, and as is demonstrated subsequently, the two marginal curves asymptotically approach the centerline of the wedgeshaped region. It is thus not clear how small the region of validity of the asymptotic, weakly nonlinear theory might be and even its qualitative importance.

The present study is an attempt to clarify the nonlinear problem. Section 2 reviews the basic model, which has continuous stratification, and the nature of the resulting linear

problem. It also describes the resonance condition that is at the heart of the instability at high wave numbers. Section 3 describes the results of weakly nonlinear theory for the continuous problem and demonstrates the possibility of nonlinear instability in some region asymptotically close to one of the stability boundaries. Section 4 describes a spatially truncated but fully nonlinear model to determine the nature of the nonlinear behavior for the most unstable wave in the center of the unstable parameter region. Section 5 summarizes the fundamental results.

\section{The model and linear theory}

The basic flow whose stability is at issue is a flow in the $x$ direction with uniform vertical shear in the $z$ direction of the form

$$
u=U_{z} z \quad 0 \leq z \leq D
$$

The current is contained in a channel whose width in the $y$ direction is $L$. The stratification is constant with a uniform buoyancy frequency $N$. The elevation of the topography is given by $h_{b}(y)$, which is a linearly decreasing function of $y$; that is,

$$
\frac{d h_{b}}{d y}=s<0,
$$


where $s$ is a constant. The critical parameter of the problem is the ratio of the bottom slope to the slope of the isopycnals. Because the flow is in geostrophic balance, this ratio can be easily shown to be

$$
Z_{T}=\frac{s}{\left.\frac{\partial z}{\partial y}\right)_{\rho}}=s N^{2} / f U_{z},
$$

where $f$ is the constant Coriolis parameter. Because in the problem of interest $Z_{T}$ is negative, it is convenient to introduce the positive parameter $\alpha_{T}=-Z_{T}>0$. Scales for velocity, horizontal lengths, vertical length, and time are chosen to be $U_{z} D, L, D$, and $L / U_{z} D$, respectively. The governing equations are the inviscid, quasi-geostrophic potential vorticity equation and associated boundary conditions. The scale for the geostrophic streamfunction is simply the velocity scale times $L$. Because the potential vorticity of the basic state is a constant, the potential vorticity remains constant in the absence of sources or sinks as in this problem. The generally finite amplitude perturbation to the basic flow is $\phi(x, y, z, t)$ and satisfies the constraint that the perturbation potential vorticity must remain zero, and hence, $\phi$ satisfies

$$
\begin{gathered}
\phi_{z z}+S\left(\frac{\partial^{2}}{\partial x^{2}}+\frac{\partial^{2}}{\partial y^{2}}\right) \phi=0, \\
S=N^{2} D^{2} / f^{2} L^{2} .
\end{gathered}
$$

In equation (4a) and what follows, subscripts generally indicate differentiation, and it should be obvious from context.

The boundary conditions for equation (4a) are that on $y=0,1$, the sidewalls contain the flow, $\phi=0$. On the horizontal boundaries, the boundary condition is that on the upper, level boundary, the vertical velocity vanishes. Hence, on $z=1$, combining the condition for conservation of density with the hydrostatic approximation and geostrophy, we obtain the condition

$$
\frac{\partial \phi_{z}}{\partial t}+\phi_{z x}-\phi_{x}+J\left(\phi, \phi_{z}\right)=0, z=1,
$$

where the Jacobian, $J$, of the two functions in parentheses is with respect to $x$ and $y$.

On the lower boundary, where the slope induces a vertical velocity, the condition of no normal flow to the boundary can be written as follows:

$$
\frac{\partial \phi_{z}}{\partial t}-\left(1+\alpha_{T}\right) \phi_{x}+J\left(\phi, \phi_{z}\right)=0 .
$$

Note that the second term in equation (5a), the advection of the perturbation by the basic flow, is missing in equation ( $5 b$ ) because the basic flow is zero there. The third term on the left-hand side of equation (5a) involves the vertical shear of the basic flow, which is unity in our scaled system. In equation (5b), that term is supplemented by the term $\alpha_{T}$ representing 
the topographic production of vertical velocity by perturbation flow in the $y$ direction up the shelf-like topography.

For the linear problem for small perturbations, the Jacobian term, of second order in the amplitude of the perturbations, can be ignored. The resulting linear equations admit wavelike solutions of the form

$$
\phi=A[\cosh \mu(z-1)+b \sinh \mu(z-1)] e^{i k(x-c t)} \sin (n \pi y)
$$

where $A$ and $b$ are arbitrary constants, $n$ is an integer, and $\mu^{2}=S\left(k^{2}+n^{2} \pi^{2}\right)$. The boundary conditions on $y=0,1$ are satisfied by equation (6), and the boundary conditions (equations $5 \mathrm{a}$ and $5 \mathrm{~b}$ ) lead to the dispersion relation for $c$, whose solution yields two roots for $c$ :

$$
c=\frac{1}{2}\left(1+\alpha_{T} \frac{\operatorname{coth} \mu}{\mu}\right) \pm\left[\frac{1}{4}\left(1+\alpha_{T} \frac{\operatorname{coth} \mu}{\mu}\right)^{2}-\left(1+\alpha_{T}\right)\left(\frac{\operatorname{coth} \mu}{\mu}-\frac{1}{\mu^{2}}\right)\right]^{1 / 2} .
$$

The perturbation becomes unstable when the radicand on the right-hand side of equation (7) becomes negative, and the condition that the radicand is just zero provides the critical curves delineating the boundaries of the unstable domain. It follows that those curves are given by

$$
\alpha_{T_{\text {crit }}}=\mu \tanh \mu-2 \tanh \mu \pm 2\left[\left(\mu \tanh \mu-\tanh ^{2} \mu\right)\left(1-\tanh ^{2} \mu\right)\right]^{1 / 2} .
$$

Figure 1(a) shows the stability diagram. Note that for negative values of $\alpha_{T}$, for which the shear is in the direction opposed to the propagation direction of topographic waves, the domain of instability is localized and has a short wave cutoff similar to the Eady problem, and that strong enough topographic slope will always stabilize the flow. On the other hand, positive values of the parameter $\alpha_{T}$, representing shears in the direction of the topographic wave propagation, are unstable for all values of $\alpha_{T}$; that is, there is no topographic slope large enough, or shears small enough, to render the flow stable (ignoring friction). The domain of instability, as can be seen in Figure 1(a), becomes increasingly narrow at large values of the total wave number $\mu$. Indeed, for large $\mu$, the boundaries of the domain delineate a thin sliver given by

$$
\alpha_{T_{c c r i t}} \approx \mu-2 \pm 4(\mu-1)^{1 / 2} e^{-\mu}+\ldots
$$

Figure 1(b) shows the growth rate for a value of $\mu=3.5$ as a function of $\alpha_{T}$ in the unstable sliver. The growth rate has its maximum near the center of the sliver at $\mu-2$ and also becomes exponentially small as a function of wave number for large $\mu$. In fact, on the centerline where $\alpha_{T}=\mu-2$, the growth rate for large $\mu$ goes like $2\left(k / \mu^{1 / 2}\right) e^{-\mu}$. The double boundary for the unstable region when $\alpha_{T}$ is positive on both branches (which coincides roughly with wave numbers greater than the Eady shortwave cutoff) has interesting 

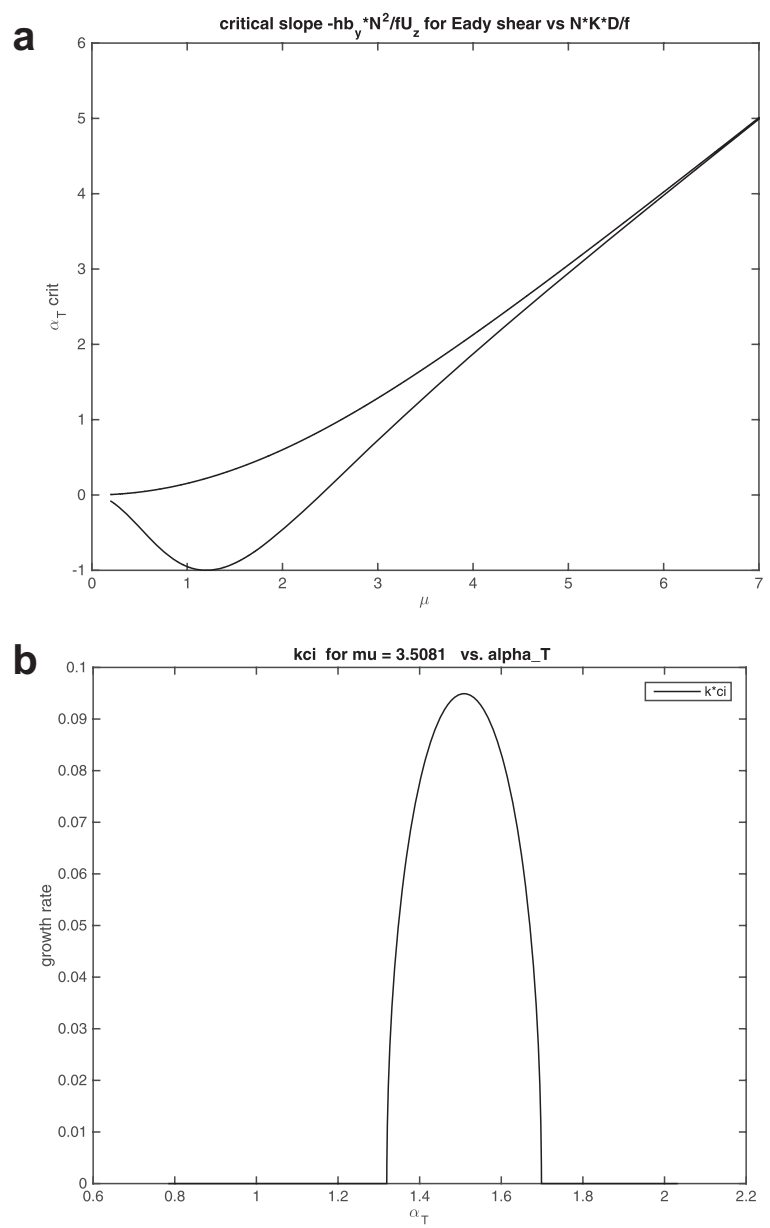

Figure 1. (a) The critical curves in the $\mu, \alpha_{T}$ plane. (b) The growth rate as a function of $\alpha_{\mathrm{T}}, \mu=3.5$.

implications, for it means that moving into the unstable region from the lower boundary shows the growth rate increasing with decreasing shear (or increasing topographic slope). This raises the possibility that the effects of nonlinearity, which we anticipate would, among other things, lower the shear as the horizontal density gradient is reduced by the instability, might increase the growth rate leading to a nonlinear enhancement of the instability instead of producing a limit to the growth.

On the other hand, a different interpretation is that the growth rate is increased the closer we get to the centerline, a locus of resonance of the perturbation with the topographic wave, which can be seen from the following standard argument (see, e.g., Vallis 2006).

If we suppose that the two horizontal boundaries are well separated, which is equivalent to examining perturbations with large $\mu$, each boundary can independently support a 
boundary-trapped, exponentially decaying wave. For the upper boundary, the wave sees the potential vorticity equivalent of the horizontal temperature gradient, and the boundary condition (equation 5a) in its linear form yields a phase speed,

$$
c_{\text {upper }}=1-1 / \mu \text {, }
$$

whereas the lower boundary supports a wave whose speed is determined by the joint effect of the topography and shear. This leads to a wave, exponentially decaying from the lower boundary, moving with phase speed,

$$
c_{\text {lower }}=\frac{1+\alpha_{T}}{\mu} .
$$

When the two boundaries are close enough so that at least a weak interaction between the two waves is possible, an instability may result and the condition that allows this is that the two speeds in equations (10a) and (10b) coincide leading to the condition that

$$
\alpha_{T}=\mu-2,
$$

which is the equation for the centerline that the two boundaries of the unstable domain approach for large wave number, consistent with equation (9). Note that in the absence of topographic slope, equation (11) would simply yield an approximation to the Eady short wave cutoff. Also, as we have seen, the growth rate increases the closer the slope approaches the value given by equation (11) (i.e., a resonance of the unstable wave with a topographic wave). Hence, an expected nonlinear alteration of the mean flow shear might possibly destabilize the flow by moving closer to the centerline by increasing $\alpha_{T}$ from the lower branch. Or the same reduction in the shear might stabilize the flow by moving the value of $\alpha_{T}$ away from the centerline (i.e., detuning the instability from the topographic resonance by moving toward the upper branch). The following two sections, devoted to the nonlinear theory, will examine this question, especially the question of detuning. First, I take up the results of weakly nonlinear theory.

\section{Weakly nonlinear theory}

For values of $\alpha_{T}$ that are near either one of the boundaries of the unstable regime, an asymptotic method based on the presumption of slow growth and small amplitude provides an analytical approach to this weakly nonlinear problem. The basic method exploits the method of multiple timescales as described in Pedlosky (1970). The key to the development is to consider the perturbations as a function of both a fast time, $t$, equal to the (inverse) real frequency of the wave along the marginal curve, and a slow time, $T$, determined by the weak growth rate. A similar approach was used in the two-layer version of this problem by Steinsaltz (1987), but for large wave numbers, the vertical scale of the perturbation in the continuous model rapidly becomes small compared with the layer depths rendering the analysis problematic if still qualitatively suggestive. 
Thus, we consider values of $\alpha_{T}$ that are near its critical value, $\alpha_{T_{C}}$, along either one of the marginal curves in the region $\mu>2$ so that $\alpha_{T}=\alpha_{T_{C}}+\Delta, \Delta \ll \alpha_{T_{C}}$. Note that $\Delta$ would be positive entering the unstable sliver from the lower curve and negative entering from the upper branch.

The equation for the geostrophic streamfunction remains equation (4) because there are no sources or sinks of potential vorticity in the interior of the fluid; Thus,

$$
\phi_{z z}+S\left(\phi_{x x}+\phi_{y y}\right)=0 .
$$

The geostrophic streamfunction is considered a function of both $t$ and $T$ so that the total streamfunction including the mean flow and the small perturbation is

$$
\psi=-z y+\varepsilon \phi(x, y, z, t, T),
$$

where $\varepsilon$ is of order $|\Delta|^{1 / 2}$. This leads to a restatement of the upper boundary condition as

$$
\frac{\partial \phi_{z}}{\partial t}+\left|\Delta^{1 / 2}\right| \frac{\partial \phi_{z}}{\partial T}+\phi_{z x}-\phi_{x}+\varepsilon J\left(\phi, \phi_{z}\right)=0 \quad z=1
$$

and the condition at $z=0$ becomes

$$
\frac{\partial \phi_{z}}{\partial t}+|\Delta|^{1 / 2} \frac{\partial \phi_{z}}{\partial T}-\left(1+\alpha_{T_{C}}+\Delta\right) \phi_{x}+\varepsilon J\left(\phi, \phi_{z}\right)=0
$$

where $\alpha_{T_{C}}$ is given by either of the two branches of equation (8). Because $\varepsilon$ is a small parameter, the perturbation streamfunction is expanded in an asymptotic power series.

$$
\phi=\phi^{(0)}+\varepsilon \phi^{(1)}+\varepsilon^{2} \phi^{(2)}+\ldots,
$$

where $\varepsilon=O\left(|\Delta|^{1 / 2}\right)$. Note that we can only expect that the expansion is asymptotic, not convergent, and so its validity is limited to a small region, whose extent is unknown, near each critical curve. What happens on the centerline, where the growth rate has its maximum, cannot be anticipated on the basis of the theory developed in this section.

The first step in carrying out the expansion in equation (15) yields the order-one problem (i.e., the problem for the neutral wave on each of the critical curves). As before, I will write the solution for the wave perturbation as

$$
\phi^{(0)}=A(T) \sin \pi y F(z) e^{i k\left(x-s_{o} t\right)}+*,
$$

where the asterisk connotes the complex conjugate of the preceding expression and

$$
\begin{gathered}
F(z)=\sinh \mu(z-1)+b_{o} \cosh \mu(z-1), \\
\mu=\left[S\left(k^{2}+\pi^{2}\right)\right]^{1 / 2} .
\end{gathered}
$$


Here, $s_{o}$ is the phase speed at one or the other of the critical curves:

$$
s_{o}=\frac{1}{2}\left(1+\alpha_{T_{c}} \frac{\operatorname{coth} \mu}{\mu}\right) .
$$

Note that in equation (16), I have chosen the smallest value of the cross-stream mode number, $n=1$, because it is the linearly most unstable mode. The order-one problem yields both $s_{o}$ and the constant

$$
b_{o}=\mu\left(1-s_{o}\right)
$$

and it is important to note that $F$ is a real function.

The order $\varepsilon$ problem is as follows:

$$
\begin{gathered}
\phi_{z z}^{(1)}+S\left(\phi_{y y}^{(1)}+\phi_{x x}^{(1)}\right)=0 ; \\
\phi_{z t}^{(1)}+\phi_{z x}^{(1)}-\phi_{x}^{(1)}=-\frac{|\Delta|^{1 / 2}}{\varepsilon} \frac{d A}{d T} \mu e^{i k\left(x-s_{o} t\right)} \sin \pi y, \quad z=1, \quad(19 \mathrm{a}, \mathrm{b}, \mathrm{c}) \\
\phi_{z t}^{(1)}-\left(1+\alpha_{T_{C}}\right) \phi_{x}^{(1)}=-\frac{|\Delta|^{1 / 2}}{\varepsilon} \frac{d A}{d T} \mu\left(\cosh \mu-b_{o} \sinh \mu\right) e^{i k\left(x-s_{o} t\right)} \sin \pi y, \quad z=0
\end{gathered}
$$

Note that there is no contribution at this order $(\varepsilon)$ from the nonlinear terms. Because the function $F(z)$ is real the Jacobian, $J\left(\phi^{(0)}, \phi_{z}^{(0)}\right)=0$.

Solutions to equation (19a) can be found in the form

$$
\phi^{(1)}=\left[a_{1} \sinh \mu(z-1)+b_{1} \cosh \mu(z-1)\right] e^{i k\left(x-s_{o} t\right)} \sin \pi y .
$$

Inserting equation (20) into the boundary conditions (equations $19 \mathrm{~b}$ and 19c) yields the following two equations:

$$
\begin{aligned}
b_{1}= & \frac{|\Delta|^{1 / 2}}{i k \varepsilon} \mu \frac{d A}{d T}+\mu\left(1-s_{o}\right) a_{1}, \\
& -s_{o} \mu\left[a_{1} \cosh \mu-b_{1} \sinh \mu\right]-\left(1+\alpha_{T_{C}}\right)\left(b_{1} \cosh \mu-a_{1} \sinh \mu\right) \\
= & -\frac{|\Delta|^{1 / 2}}{i k \varepsilon} \frac{d A}{d T}\left(\cosh \mu-b_{1} \sinh \mu\right) .
\end{aligned}
$$

With some algebra, and using the dispersion relations for $s_{o}$, it can be shown that the two equations are redundant. As in Pedlosky (1970), this is related to the necessary condition for instability of the system. Because the equations are redundant, one of $a_{1}, b_{1}$ can be arbitrarily chosen to be zero. Here, we choose $a_{1}$ to be zero leading to the following solution at this order:

$$
\phi^{(1)}=\frac{\mu}{i k} \frac{|\Delta|^{1 / 2}}{\varepsilon} \frac{d A}{d T} \cosh \mu(z-1) e^{i k\left(x-s_{o} t\right)} \sin \pi y+*+\Phi^{(1)}(y, z, T),
$$


which represents a phase shift in the wave proportional to the slow time evolution of the wave amplitude. We have also added, at this order $\left(\Phi^{(1)}\right)$, a streamfunction representing a correction to the zonal flow that needs to be determined at the next order.

It is useful to think of the wavy part of the solution as follows:

$$
\begin{gathered}
\phi_{12}=e^{i k\left(x-s_{o} t\right)} \sin \pi y G(z, T), \\
G=A(T) F(z)+\varepsilon \frac{\mu}{i k} \frac{|\Delta|^{1 / 2}}{\varepsilon} \frac{d A}{d T} \cosh \mu(z-1) .
\end{gathered}
$$

The important point is that both $G$ and it complex conjugate are solutions of the equation for the vertical structure of the wave perturbation,

$$
G_{z z}-\mu^{2} G=0
$$

It therefore follows that the Wronskian of the function $G$ and its complex conjugate is independent of $z$; that is,

$$
\frac{d}{d z}\left[G G_{z}^{*}-G_{z} G\right]=0
$$

This has importance because the Jacobian of $\phi$ and $\phi_{z}$ is proportional to the Wronskian. So, just as in the Eady problem, the heat flux is independent of $z$ within the fluid and, in particular, is the same at both boundaries, $z=0,1$. This has implications for the structure of the correction to the mean flow, and it also implies that the forcing term for that correction by the growing wave field is the same at both boundaries. Indeed a straight forward calculation shows that at order $\varepsilon^{2}=O(\Delta)$, the part of the nonlinear forcing on the boundary independent of $x$ leads to the condition for the mean flow correction,

$$
\frac{\partial \Phi_{z}^{(1)}}{\partial T}=-\mu^{2} \pi \frac{|\Delta|^{1 / 2}}{\varepsilon} \frac{d|A|^{2}}{d T} \sin 2 \pi y, \quad z=0,1,
$$

whereas in the fluid interior,

$$
\Phi_{z z}^{(1)}+\Phi_{y y}^{(1)}=0
$$

Integrating equation (26) with respect to time yields as a boundary condition,

$$
\Phi_{z}^{(1)}=-\mu^{2} \pi\left(|A|^{2}-\left|A_{o}\right|^{2}\right) \sin 2 \pi y, \quad z=0,1,
$$

where $A_{o}$ is the initial value of the $O(1)$ wave amplitude.

It follows that the structure of the mean flow correction to the vertical shear will be symmetric about the midpoint in $z$, whereas the correction to the velocity will be antisymmetric (i.e., purely baroclinic).

The boundary condition for the mean flow correction at $y=0,1$ is the absence of a velocity normal to the boundary. Because $\Phi^{(1)}$ is independent of $x$, it has no geostrophic 
velocity in the $y$ direction, but it does have an ageostrophic velocity. To ensure that the ageostrophic $y$ velocity vanishes, the condition

$$
\frac{\partial \Phi_{y}^{(1)}}{\partial T}=0, \quad y=0,1
$$

must be satisfied (Pedlosky 1970). This leads to a solution for the mean flow correction in terms of the wave amplitude,

$$
\begin{gathered}
\Phi^{(1)}(y, z, T)=\sum_{j=1}^{J_{\max }} \Phi_{j}(T) \cos j \pi y\left[\cosh m_{j} z-\cosh m_{j}(z-1)\right], \\
\Phi_{j}=\frac{\mu^{2}\left[|A|^{2}-\left|A_{o}\right|^{2}\right]}{m_{j} \sinh m_{j}}\left(\frac{4}{4-j^{2}}\right)\left(1-(-1)^{j}\right), \quad m_{j}=S^{1 / 2} j \pi . \quad \text { (29a, b, c) }
\end{gathered}
$$

The upper limit of the sum in equation (29a) is, in principle, infinite, but the rapidly convergent series is accurate for a value of $J_{\max }$ of order 10 . Note that all even $j$ terms in the sum are zero. From equations (27b) and (29b), it is easy to verify that when the wave amplitude exceeds its initial value, the shear of the mean flow is reduced in a region around the center of the channel at $y=1 / 2$, where the order-one eigenfunction has its maximum.

With the $O\left(\varepsilon^{2}\right)$ correction to the zonal flow determined by equation $(29 \mathrm{a}, \mathrm{b}, \mathrm{c})$, the next step is to consider the wave field at the same order (i.e., $\left.\phi^{(2)}\right)$. The second-order correction to the wave disturbance also satisfies equation (12), so the solution can be written as follows:

$$
\phi^{(2)}=A_{2}\left[\sinh \mu(z-1)+b_{2} \cosh \mu(z-1)\right] e^{i k\left(x-s_{o} t\right)} \sin \pi y
$$

with boundary conditions

$$
\begin{gathered}
\phi_{z t}^{(2)}+\phi_{z x}^{(2)}-\phi_{x}^{(2)}=-\frac{|\Delta|^{1 / 2}}{\varepsilon} \frac{\partial \phi_{z}^{(1)}}{\partial T}-\phi_{x}^{(0)} \Phi_{z y}^{(1)}+\phi_{z x}^{(0)} \Phi_{y}^{(1)} \quad z=1, \\
\phi_{z t}^{(2)}-\left(1+\alpha_{T_{c r i t}}\right) \phi_{x}^{(2)}=-\frac{|\Delta|^{1 / 2}}{\varepsilon} \frac{\partial \phi_{z}^{(1)}}{\partial T}-\phi_{x}^{(0)} \Phi_{z y}^{(1)}+\phi_{z x}^{(0)} \Phi_{y}^{(1)}+\frac{\Delta}{\varepsilon^{2}} \quad z=0 .
\end{gathered}
$$

Note that it is at this order that the supercriticality of the wave (i.e., its parametric distance from its neutral curve) enters the problem for the first time. To find the amplitude evolution equation for the amplitude of the order-one wave field, two algebraically intense steps must be carried out. The right-hand sides of equations (31a) and (31b) must first be projected onto the horizontal spatial structure of the $O(1)$ wave field (i.e., $e^{i k\left(x-s_{o} t\right)} \sin \pi y$ ). I will refer to those projections as $R_{1}$ for the projection at the upper boundary at $z=1$ and $R_{0}$ for that projection at $z=0$. Secondly, the condition that the projected forcing does not produce a resonance with the linear operator of the left-hand side invalidating our basic expansion (equation 15) can be shown, with a fair amount of algebra, to be simply

$$
R_{0}+R_{1}\left[s_{o} \mu \sinh \mu-\left(1+\alpha_{T_{\text {crit }}}\right) \cosh \mu\right]=0 .
$$


where $s_{o}$ and $\alpha_{T_{c r i t}}$ are given by equations (17c) and (8), respectively. Note again that there are two marginal curves given in equation (8), and our analysis refers to both. Collecting all the terms implied by equation (32) yields the following second-order differential equation for the wave amplitude $A$ from which the correction to the mean flow follows as well. After really considerable algebra, we obtain

$$
\frac{1}{k^{2}} \frac{d^{2} A}{d t^{2}}-\frac{\Delta}{|\Delta|} A\left(b_{o} \cosh \mu-\sinh \mu\right)+\frac{\varepsilon^{2}}{|\Delta|} N_{L} A\left(|A|^{2}-\left|A_{0}\right|^{2}\right)=0 .
$$

The form of the amplitude equation is standard and for the baroclinic problem was derived in Pedlosky (1970), and a discussion is given there of the behavior of the solutions. Suffice it to say that the second derivative in time merely reflects the inviscid, adiabatic nature of the dynamics rendering the solution reversible in time. The second term is just the square of the growth rate (divided by $k$ ) given by linear theory near the marginal curve. The last term represents the nonlinear interaction of the developing wave with the altered zonal flow. This term, cubic in the amplitude, determines the longtime behavior of the wave. The principal result is the Landau coefficient, $N_{L}$. If $N_{L}$ is positive, the behavior is oscillatory with a period and amplitude depending on $N_{L}$ and the linear growth rate. If $N_{L}$ is negative, the nonlinearity, instead of putting a ceiling on the wave growth, will instead accelerate the growth, and the amplitude will continue to grow until the amplitude is so large that the weakly nonlinear theory loses validity.

The calculation of $N_{L}$ follows directly from the steps outlined previously. After considerable calculation, we obtain the following:

$$
\begin{gathered}
N_{L}=N_{1}+N_{2} \\
N_{1}=\mu^{2} \pi^{2}\left[b_{o} \cosh \mu-\sinh \mu+b_{o}\left(s_{o} \mu \sinh \mu-\left(1+\alpha_{T_{c r i t}}\right) \cosh \mu\right]\right. \\
N_{2}=16 \mu^{3}\left[\cosh \mu-b_{o} \sinh \mu-\left(s_{o} \mu \sinh \mu+\left(1+\alpha_{T_{c r i t}}\right) \cosh \mu\right]\right. \\
\times \sum_{j=1}^{J_{\max }} \frac{\left(1-(-1)^{j}\right)^{2}\left(\cosh m_{j}-1\right)}{m_{j} \sinh m_{j}}
\end{gathered}
$$

The Landau constant $N_{L}$ is shown in Figure 2 as a function of $\mu$. In Figure 2(a), the Landau constant for the upper branch of the marginal stability curve, which yields the upper boundary of the unstable domain, is positive, shown as the solid line. As anticipated, the effect of the interaction of the wave with the altered mean current is stabilizing, and a nonlinear oscillation results. For the weakly nonlinear theory in the region near the lower marginal stability curve, the Landau constant is given by the dashed curve and is negative, and so the nonlinear effects are destabilizing. A qualitatively similar result for the two-layer model was found by Steinsaltz (1987), but the behavior, as a function of wave number, is different. The decrease of the nonlinear interaction as $\mu$ increases is not found in the two-layer model; which is not surprising given the inability of the layer model to accurately reflect the vertical structure for large $\mu$. Figure 2(b) shows the two components $N_{1}$ and $N_{2}$ 

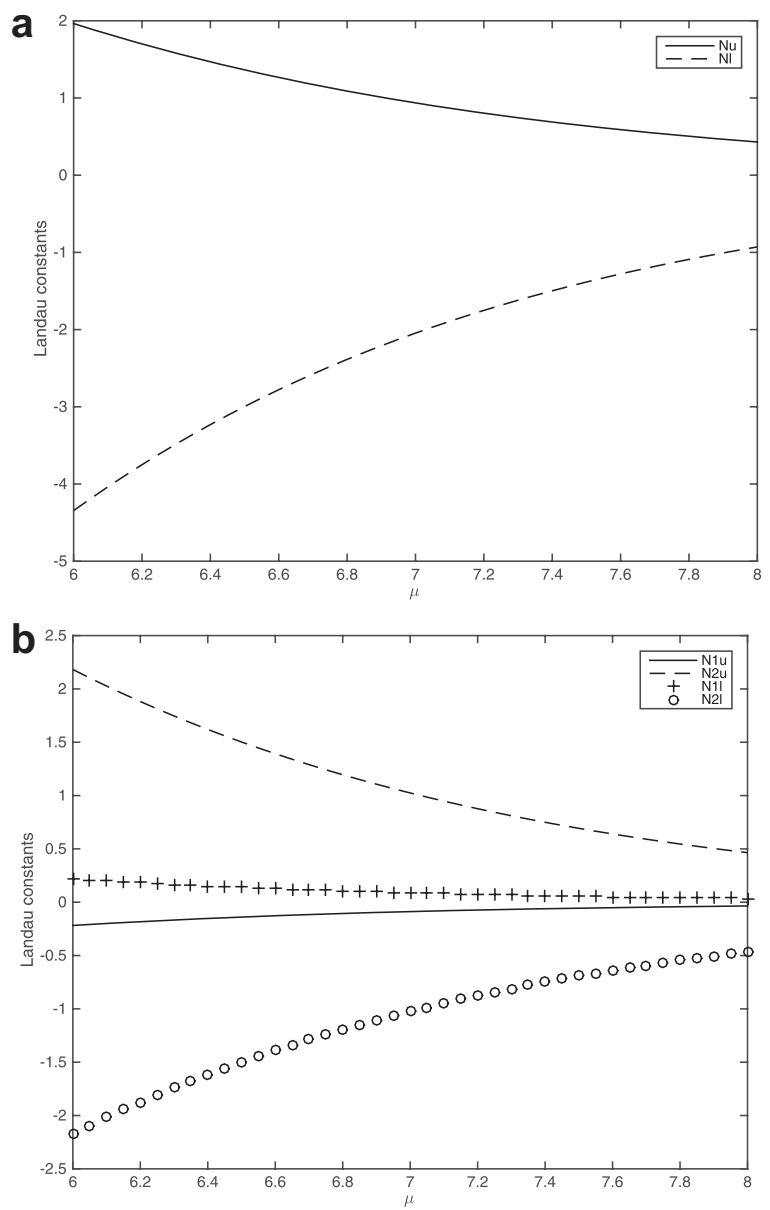

Figure 2. (a) The Landau constant, $N_{L}$, as a function of $\mu$ for both branches of the stability curve. (b) The two components of each of the Landau constants. The dotted curves relate to the contribution to the Landau constant of the nonlinear differential advection on the top and bottom surfaces, whereas the solid lines are due to the change in the shear on those surfaces.

of the Landau constant. The two components for the upper branch include the component due to the alteration of the vertical shear on the boundary given by the solid curve, which is negative, and the contribution due to the change in the zonal flow advection on the boundary, which is given by the dashed line and is the dominant contribution and stabilizing. The two components on the lower branch have the opposite signs and are given by the plus sign for the shear contribution and the open circle for the advection, and this result is consistent with Steinsaltz (1987), although the behavior as a function of $\mu$ is quantitatively different as we expect given the limitation of the two-layer model. Of course, the two effects are 
due to the same shear reduction that reduces the zonal velocity at the upper boundary and increases it at the lower boundary. It is possible to rationalize this effect as a detuning of the perturbation and the topographic wave as described by equations (10a) and (11b).

For the moment, to aid our interpretation let us restore the explicit representation of the shear and the velocity fields as trace constants. This would give rise, for large wave numbers, to a form of equations (10a) and (10b) as follows:

$$
\begin{gathered}
c_{\text {upper }}=U(1)-U_{z} / \mu, \\
c_{\text {lower }}=U(0)+\left(U_{z}+\alpha_{T}\right) / \mu,
\end{gathered}
$$

ignoring small exponential terms in wave number. Equating the two expressions for the phase speeds at large values of $\mu$, and recalling that $\alpha_{T}$ is of order $\mu$, yields the approximate condition that relates the position of the critical curves to the effect of the differential advection of temperature at the boundaries. It dominates that of the shear, so that asymptotically,

$$
\alpha_{T_{\text {crit }}} \simeq \mu(U(1)-U(0))
$$

Because the effects of nonlinearity are to lower the velocity at the upper boundary and increase it at the lower boundary, the effect of the differential nonlinear correction to the differential advection would be to lower each of the critical curves. For a fixed value of the slope (i.e., for fixed $\alpha_{T}$ ), a point near enough to the linear theory's upper branch would find itself in the stable region (i.e., detuned from the instability condition), whereas a slightly unstable wave near the lower boundary would, on the contrary, find itself deeper into the unstable region. These opposing effects of the shear reduction by the nonlinearity explain the behavior of the Landau constants in Figure 2(b).

The more pressing question is what happens at the value of $\alpha_{T}$ associated with the peak of the growth rate, for example, at $\mu=3.5$ in Figure 1(b). Note that this corresponds almost exactly to a value of $\alpha_{T}=\mu-2$. The Landau constant is positive at one end of the unstable interval on the upper branch and negative on the lower branch. Weakly nonlinear theory gives no guidance as to what to expect for the most unstable wave at the center of the unstable region. In the next section, this question is examined using a nonasymptotic truncated model.

\section{A nonlinear truncated model}

To consider a nonlinear model without the use and limitations of weakly nonlinear theory, I will consider a model consisting of a single wave interacting with a variable mean flow. The growth rates in the region of interest are small, and the amplitudes expected, assuming there is nonlinear equilibration, are predicted to be small, so a truncation of the form of the solution seems plausible. However, the assumption that the parameter $\alpha_{T}$ is asymptotically close to one of the marginal curves is no longer valid. A consequence of this approach is that the simple relation between the wave amplitude variation with time and the production 
of alterations to the mean zonal current as in equation (26) is no longer valid. Such simple results require asymptotically small perturbations such that the basic wave dynamics remain linear. That assumption is here abandoned.

Nevertheless, it is still true that the potential vorticity in the wave field and in the zonal mean current remains constant. Hence, both the wave field and the mean flow correction continue to satisfy equation (12). For the wave field, it is convenient to write the solution as

$$
\phi=[A(t) \cosh \mu z+B(t) \cosh \mu(z-1)] \sin \pi y e^{i k x}+*,
$$

and the zonal flow correction, a function of only $y$ and $z$, is

$$
\Phi(y, z, t)=\sum_{j=1}^{J_{\max }} \Phi_{j}(t)\left(\cosh m_{j}(z-1)-\cosh m_{j} z\right) \cos j \pi y .
$$

In equations (37) and (38), the symbols have the same meaning as in Section 3.

In equation (38), I have exploited the fact that the condition that the wave field has constant potential vorticity implies that the nonlinear forcing terms will be the same on both horizontal boundaries as in the weakly nonlinear problem, and the structure in equation (38) anticipates that result. Indeed using equations (5a) and (5b), the equation for the zonal mean easily leads to the equation for $\Phi_{j}$, namely,

$$
\frac{d \Phi_{j}}{d t}=i \frac{4 k \mu}{m_{j} \sinh m_{j}} \sinh \mu\left(A B^{*}-A^{*} B\right) \frac{\left(1-(-1)^{j}\right)}{j^{2}-4} .
$$

The boundary conditions on $z=0$ and 1 involve the projection on $\sin \pi y$ of the interaction of the wave and the correction to the zonal flow leading to the following two equations:

$$
\begin{aligned}
& \frac{\partial A}{\partial t} \mu \sinh \mu+i k A \mu \sinh \mu-i k(A \cosh \mu+B) \\
& \quad+i k(A \cosh \mu+B) 2 \int_{0}^{1} \Phi_{z y} \sin ^{2} \pi y d y-i k 2 \int_{0}^{1} \Phi_{y} \sin ^{2} \pi y d y=0,
\end{aligned}
$$

and

$$
\begin{aligned}
& \frac{\partial B}{\partial t} \mu \sinh \mu+i k\left(1+\alpha_{T}\right)(A+B \cosh \mu)\left(1-2 \int_{0}^{1} \Phi_{z y} \sin ^{2} \pi y d y\right) \\
& \quad-i k \mu B \sinh \mu 2 \int_{0}^{1} \Phi_{y} \sin ^{2} \pi y d y=0 .
\end{aligned}
$$


Carrying out the integrals in equations (40a) and (40b) and using equation (38) yields the final equations for $A$ and $B$, namely,

$$
\begin{aligned}
& \frac{d A}{d t} \mu \sinh \mu+i k A \mu \sinh \mu-i k(A \cosh \mu+B) \\
& \quad-i k(A \cosh \mu+B) S_{1}+i k A \mu \sinh \mu S_{2}=0, \\
& \frac{d B}{d t} \mu \sinh \mu+i k\left(1+\alpha_{T}\right)(A+B \cosh \mu)\left(1+S_{1}\right)-i k B \mu \sinh \mu S_{2}=0 .
\end{aligned}
$$

$S_{1}$ and $S_{2}$ are sums involving the mean flow corrections and are

$$
\begin{gathered}
S_{1}=4 \sum_{j=1}^{J_{\max }} \Psi_{j} \frac{\left(1-(-1)^{j}\right)}{j^{2}-4}, \\
S_{2}=4 \sum_{j=1}^{J_{\max }} \Psi_{j} \frac{\left(1-(-1)^{j}\right)}{j^{2}-4} \frac{\cosh m_{j}-1}{m_{j} \sinh m_{j}},
\end{gathered}
$$

and

$$
\Psi_{j}=\Phi_{j} m_{j} \sinh m_{j}
$$

Equations (39) and (41a, b) with (42a, b, c) complete the nonlinear truncated model. In most of the calculations shown, nine terms are kept in the sums in equation (42a, b), and adding more terms does not perceptibly change the results.

I will make one final alteration in this system of equations. One can anticipate that in the narrow parameter region between the two marginal curves, the real part of the phase speed will be close to what is given by linear theory and not much altered by the presence of the slower evolution due to the relatively weak instability and its accompanying nonlinearity. To keep the representations of the solutions from being complicated by this relatively rapid linear oscillation, I will make the transformation

$$
A=A_{o} e^{-i k s_{o} t}, \quad B=B_{o} e^{-i k s_{o} t}
$$

and present the results for $A_{o}$ and $B_{o}$. The alterations of equations (41a) and (41b) are obvious and not shown here for the sake of brevity. Note that the form of equation (39) is unchanged if written in terms of the new amplitudes $A_{o}$ and $B_{o}$.

Figure 3 shows the amplitude evolution of both the real and imaginary parts of $A$ and $B$ for $\mu=3.5$, the case discussed in the previous section. However, in Figure 3 the value of $\alpha_{T}$ is now chosen to be 1.5, which, as seen in Figure 1(b), is the value associated with the maximum of the linear growth rate.

Figure 3(a) shows the evolution of the linearized system (i.e., the system ignoring the nonlinear interactions at the maximum growth rate). The evolution is plotted against $k t$ so 
a

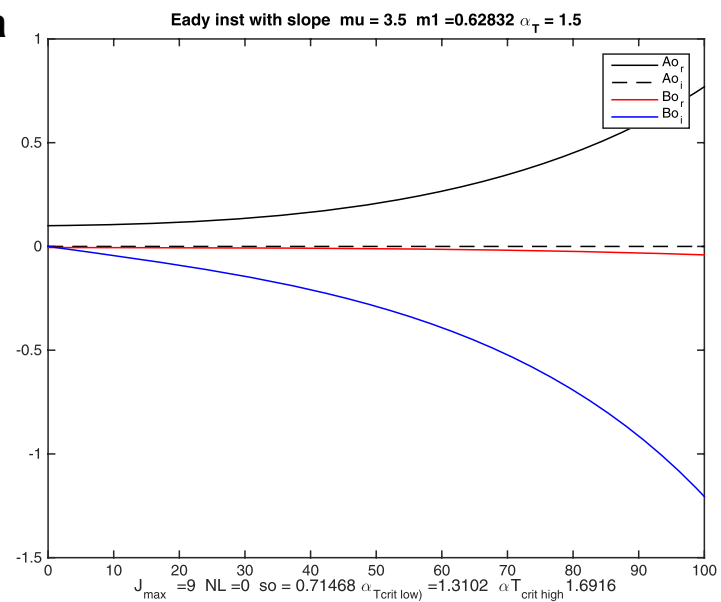

b

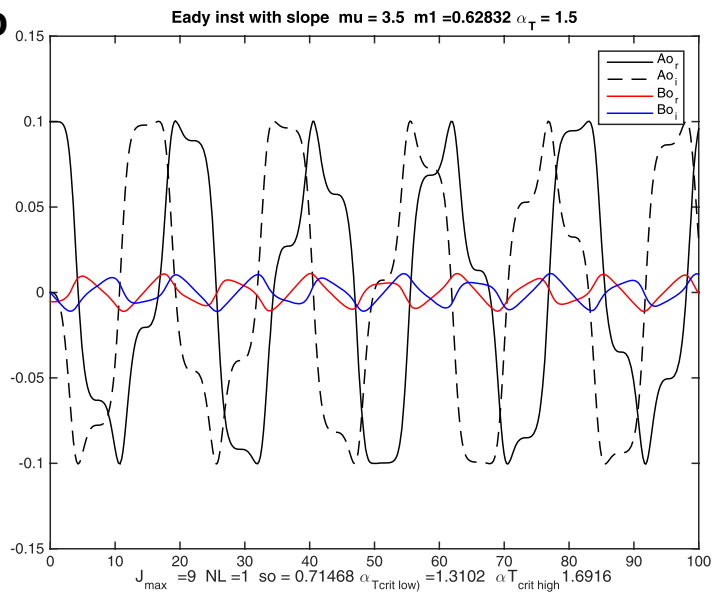

C

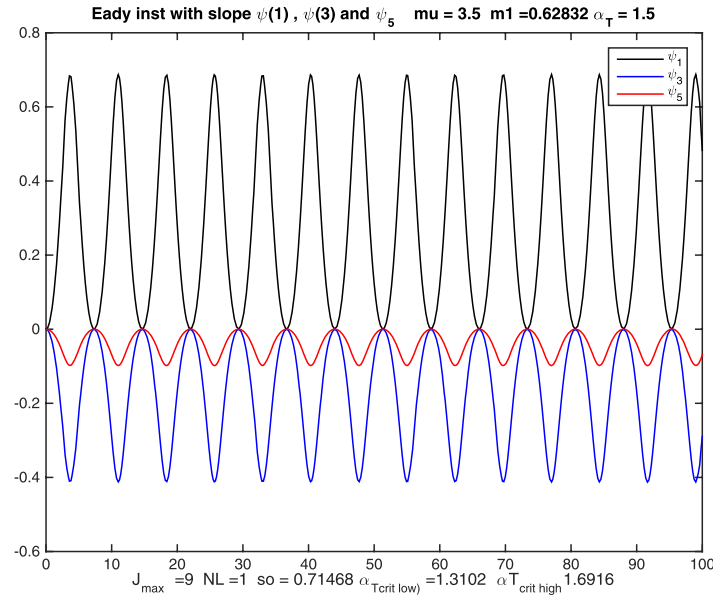

Figure 3. (a) The time evolution of the linear system at $\alpha_{T}=1.5, \mu=3.5$ corresponding to the maximum growth rate for that value of $\mu$. The real part of $A$ is shown as the black solid line, and its imaginary part is black and dashed; the real part of $B$ is red, and the imaginary part is blue. (b) The nonlinear case. (c) For the nonlinear case, the time history of the first three amplitudes of the cosine expansion of the mean field streamfunction are shown. 
that $k$ does not appear in the final equations. Note that $k^{2} / \pi^{2}=\left(\mu^{2} / m_{1}^{2}-1\right)$ so that its value is implicit given $m_{1}$ and $\mu$.

The exponential growth is evident. Figure 3(b) shows the evolution of the amplitudes when the interaction of the wave with the altered mean flow is included and the exponential growth is halted and a nonlinear oscillation of the amplitude is evident. The first three components of $\Psi_{j}(t)$ are shown in Figure 3(c). They, too, are periodic, and the signs are such that the first two, which are dominant, represent a decrease of the shear in the center of the channel where the eigenfunction is a maximum. We see that the linearly most unstable perturbation is definitely stabilized by the nonlinear interaction between the wave and the mean flow.

This raises the question of the extent of the region of validity of the asymptotic, weakly nonlinear theory near the lower branch where the weakly nonlinear theory predicts that nonlinear effects would not stabilize the perturbations. For $\mu=3.5$, the lower branch corresponds to a value of $\alpha_{T}=1.302$. Figure 4(a) shows the result of the nonlinear truncated model at $\alpha_{T}=1.311$ (i.e., only slightly into the unstable region). Because the growth rates and amplitudes are so small in this region, the calculation is carried out until $k t=4,000$. It appears that the effects of nonlinearity are still mildly stabilizing compared with the linear calculation, not shown here, which yields evident exponential growth after $k t=3,000$. Here there is still growth, but it is weaker, at least to this point in time. For a very slightly larger value of $\alpha_{T}=1.34$, the nonlinearity is definitely stabilizing as shown in Figure 4(b). Hence, around the lower branch the region of validity of the weakly nonlinear theory seems to be very limited, and the general qualitative result would appear to indicate that the interaction with the altered mean flow is generally stabilizing. Calculations around the upper branch reveal, as expected, that the interaction is stabilizing there as well.

Nevertheless, in all cases the nonlinearity does not quench the growth, and finite amplitude perturbations, at least in inviscid theory, persist into regions in which the flow would be definitely stable in the absence of bottom topography. Again, this occurs when the direction of the shear is identical to the direction of the propagation of topographic waves when no shear is present.

\section{Summary and discussion}

The apparently paradoxical result that the addition of what is generally considered to be a stabilizing agent for quasi-geostrophic instabilities, namely, uniform bottom slope, can actually destabilize a baroclinic current is shown to involve a type of resonance of the current shear with topographic waves. The presence of the shear produces waves on both boundaries, understandable because the horizontal density gradients responsible for the shear can act as surface potential vorticity gradients. Indeed, in layer models, the identification is exact. When these shear-induced waves resonate with the topographic wave, an instability well beyond the short wave cutoff of the Eady problem results. Our discussion has revealed that weakly nonlinear theory, limited to asymptotically small regions near the marginal curves, 


\section{a}

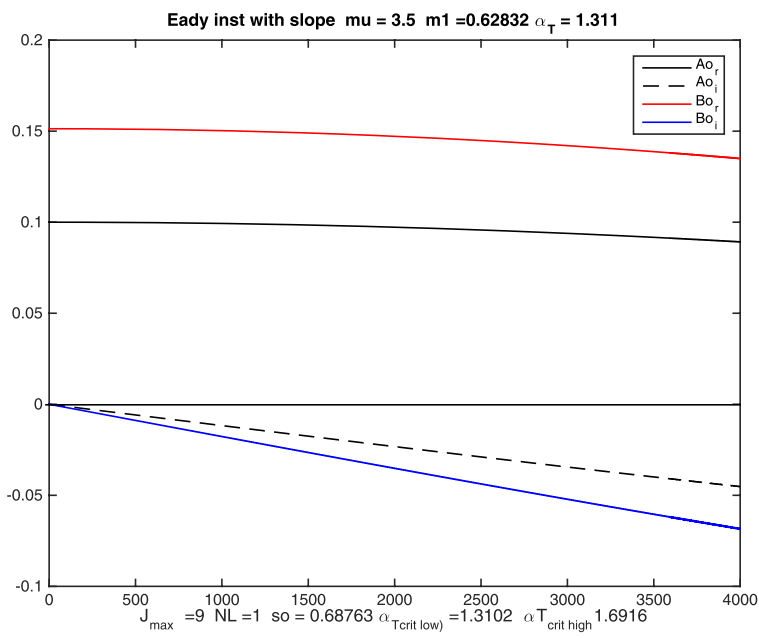

b

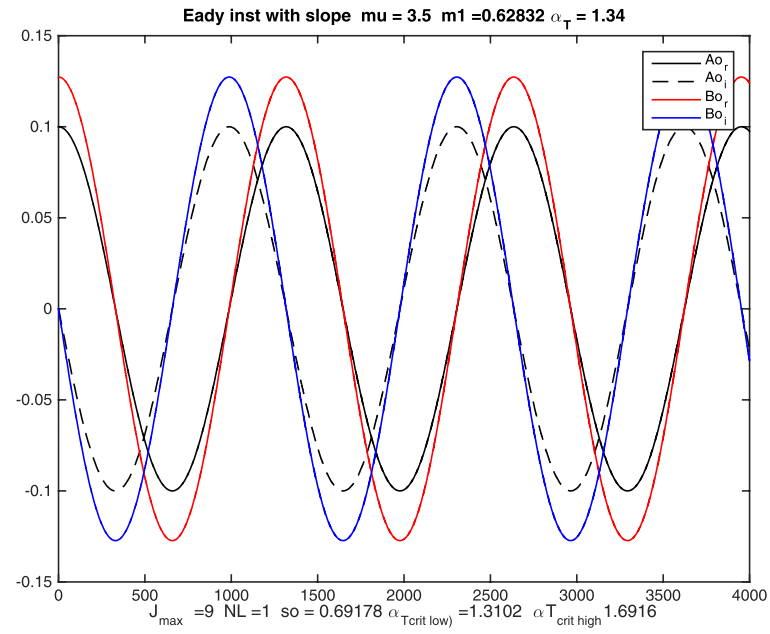

Figure 4. (a) The evolution of the perturbation amplitudes at $\alpha_{T}=1.311$, slightly into the unstable region from the lower branch. The effects of nonlinearity alter the linear evolution (which is exponential) but do not stabilize the perturbation. (b) The same calculation for $\alpha_{T}=1.34$ for which it is clear that the interaction with the mean flow is stabilizing.

can be qualitatively misleading. The nonlinear destabilization resulting near the lower of the two branches of the stability marginal curve is limited to a very small region. For more robustly unstable waves near the center of the region, the nonlinearity has been shown to be stabilizing.

Because the linear growth rates in this extended domain are small and the wave numbers are large, it is natural to wonder whether the effects of friction render these results of small 
importance in spite of their intrinsic interest. However, if the friction is primarily the result of a bottom Ekman layer type of interaction, the frictional effect relative to the inertial effects driving the instability actually diminishes as the wave number increases (Pedlosky 1987).

Acknowledgments. The author is grateful to Dr. Ken Brink for helpful discussions on this problem.

\section{REFERENCES}

Barth, J. A. 1989. Stability of a coastal upwelling front: 2. Model results and a comparison with observations. J. Geophys. Res.: Oceans, 94, 10857-10883.

Blumsack, S. L., and P. J. Gierasch. 1972. Mars: The effects of topography on baroclinic instability. J. Atmos. Sci., 29, 1081-1089.

Brink, K. H. 2012. Baroclinic instability of an idealized tidal mixing front. J. Mar. Res., 70, 661-688. Eady, E. T. 1949. Long waves and cyclone waves. Tellus, 1, 33-52.

Pedlosky, J. 1970. Finite-amplitude baroclinic waves. J. Atmos. Sci., 27, 15-30.

Pedlosky, J. 1987. Geophysical Fluid Dynamics. New York: Springer-Verlag, 710 pp.

Steinsaltz, D. 1987. Instability of baroclinic waves with bottom slope. J. Phys. Ocean, 17, 2343-2350.

Vallis, G. K. 2006. Atmospheric and Oceanic Fluid Dynamics. Cambridge: Cambridge University Press, $745 \mathrm{pp}$.

Received: 9 November 2015; revised: 2 January 2016. 